\title{
Leukemia inhibitory factor regulates microvessel density by modulating oxygen- dependent VEGF expression in mice
}

\author{
Yoshiaki Kubota, ${ }^{1}$ Masanori Hirashima, ${ }^{2}$ Kazuo Kishi, ${ }^{3}$ Colin L. Stewart, ${ }^{4}$ and Toshio Suda ${ }^{1}$ \\ 1Department of Cell Differentiation, Sakaguchi Laboratory, School of Medicine, Keio University, Shinjuku-ku, Tokyo, Japan. ²Division of Molecular Biology, \\ Department of Biochemistry and Molecular Biology, Kobe University Graduate School of Medicine, Kobe, Hyogo, Japan. ${ }^{3}$ Department of Plastic and \\ Reconstructive Surgery, School of Medicine, Keio University, Shinjuku-ku, Tokyo, Japan. ${ }^{4}$ Institute for Medical Biology, Singapore, Republic of Singapore.
}

\begin{abstract}
To meet tissue requirements for oxygen, capillaries must be properly distributed without excess or shortage. In this process, tissue oxygen concentration is well known to determine capillary density via the hypoxiainduced cascade, in which HIFs and VEGF play key roles. However, some additional mechanisms modulating this cascade are suggested to be involved in precise capillary network formation. Here, we showed that leukemia inhibitory factor (LIF) was predominantly expressed in developing endothelium, while its receptor was expressed in surrounding cells such as retinal astrocytes. The retinas of $\mathrm{Lif}^{/-}$mice displayed increased microvessel density accompanied by sustained tip cell activity, due to increased VEGF expression by astrocytes in the vascularized area. Lif $^{/-}$mice resisted hyperoxygen insult in the oxygen-induced retinopathy model, whereas they paradoxically had increased numbers of neovascular tufts. In an in vitro study, LIF inhibited hypoxia-induced VEGF expression and proliferation in cultured astrocytes. Lif ${ }^{\prime-}$ mice also exhibited similarly increased microvessel density and upregulated VEGF in various tissues outside the retina. Together, these findings suggest that tissues and advancing vasculature communicate to ensure adequate vascularization using LIF as well as oxygen, which suggests a new strategy for antiangiogenic therapy in human diseases such as diabetic retinopathy and cancer.
\end{abstract}

\section{Introduction}

In mammalian development, a vascular network is formed throughout the body to meet the tissue requirements for oxygen and nutrients (1). A hypoxia-induced cascade is widely known as the most critical mechanism underlying this process. HIFs activated in hypoxia-sensing cells trigger the expression of several genes, including that encoding VEGF (2). A negative feedback mechanism has also been implicated, in which the vasculature itself acts against such proangiogenic events triggered by hypoxia (3).

In sprouting angiogenesis, specialized endothelial tip cells lead the outgrowth of blood vessels (4). The tip cells migrate, sense, and respond to guidance cues provided by soluble, cell-bound, or matrix-bound ligands, such as VEGF (4). Retinal vascular development is controlled by interactions among neurons, astrocytes, and endothelial cells (5-7). Neurons secrete PDGF-A to stimulate proliferation of astrocytes (5), which in turn promote vascular growth by secreting $\operatorname{VEGF}(8,9)$. The directed extension of endothelial filopodia is mediated by VEGFR2 and is dependent on the correct spatial distribution of heparin-binding VEGF within the retina (4, 10). In contrast, as a feedback signal from the vasculature, oxygen from the blood may trigger astrocyte differentiation by inducing cessation of cell division, upregulation of glial fibrillary acidic protein (GFAP), and downregulation of VEGF (3), counteracting the hypoxia-induced proangiogenic state of astrocytes (11).

Nonstandard abbreviations used: FACS, fluorescence-activated cell sorting; FOV, field(s) of view; GFAP, glial fibrillary acidic protein; IHC, immunohistochemistry; ISH, in situ hybridization; LIF, leukemia inhibitory factor; LIFR, LIF receptor; NVT, neovascular tuft; OIR, oxygen-induced retinopathy.

Conflict of interest: The authors have declared that no conflict of interest exists. Citation for this article: J. Clin. Invest. 118:2393-2403 (2008). doi:10.1172/JCI34882.
Leukemia inhibitory factor (LIF) is widely known as a critical molecule for the maintenance of ES cells in culture (12). In addition, several studies have shown a physiological role of LIF in blastocyst implantation (13) and in inflammation (14). Although exogenously administered or overexpressed LIF has been reported to inhibit angiogenesis $(15,16)$, to our knowledge, neither the physiological role of LIF in angiogenesis nor the precise underlying mechanisms in the vasoinhibitory effect is known to date. In this study, we showed that LIF was predominantly expressed in developing endothelium, and the LIF receptor (LIFR) was expressed in surrounding cells to be vascularized, such as retinal astrocytes. Using Lif ${ }^{\prime-}$ mice, we showed that tissues and the advancing vasculature communicated to ensure adequate vascularization, utilizing the LIF signal cooperatively with oxygen.

\section{Results}

LIF is predominantly expressed in endothelial cells, and LIFR is expressed in surrounding cells during vascular development. Our first objective was to determine the expression pattern of LIF and LIFR in the mouse retina and other tissues during embryonic and postnatal development. In experiments using retinal cells sorted by fluorescence-activated cell sorting (FACS; Figure 1, $A$ and $B$ ), we found that LIF was predominantly expressed in endothelial cells and LIFR was expressed in astrocytes (Figure 1C). Immunohistochemistry (IHC) and in situ hybridization (ISH) of whole-mount P4 retinas showed that LIF was expressed in endothelial cells (Figure 1, D-H), and LIFR was abundantly expressed in astrocytes, although the surrounding neurons also expressed LIFR (Figure 1, I-R). This typical ligand/receptor relationship between endothelium and surrounding cells was 


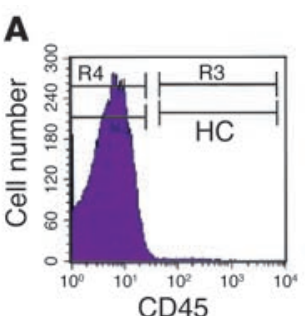

B

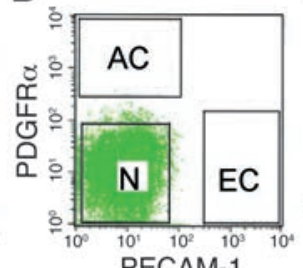

PECAM-1
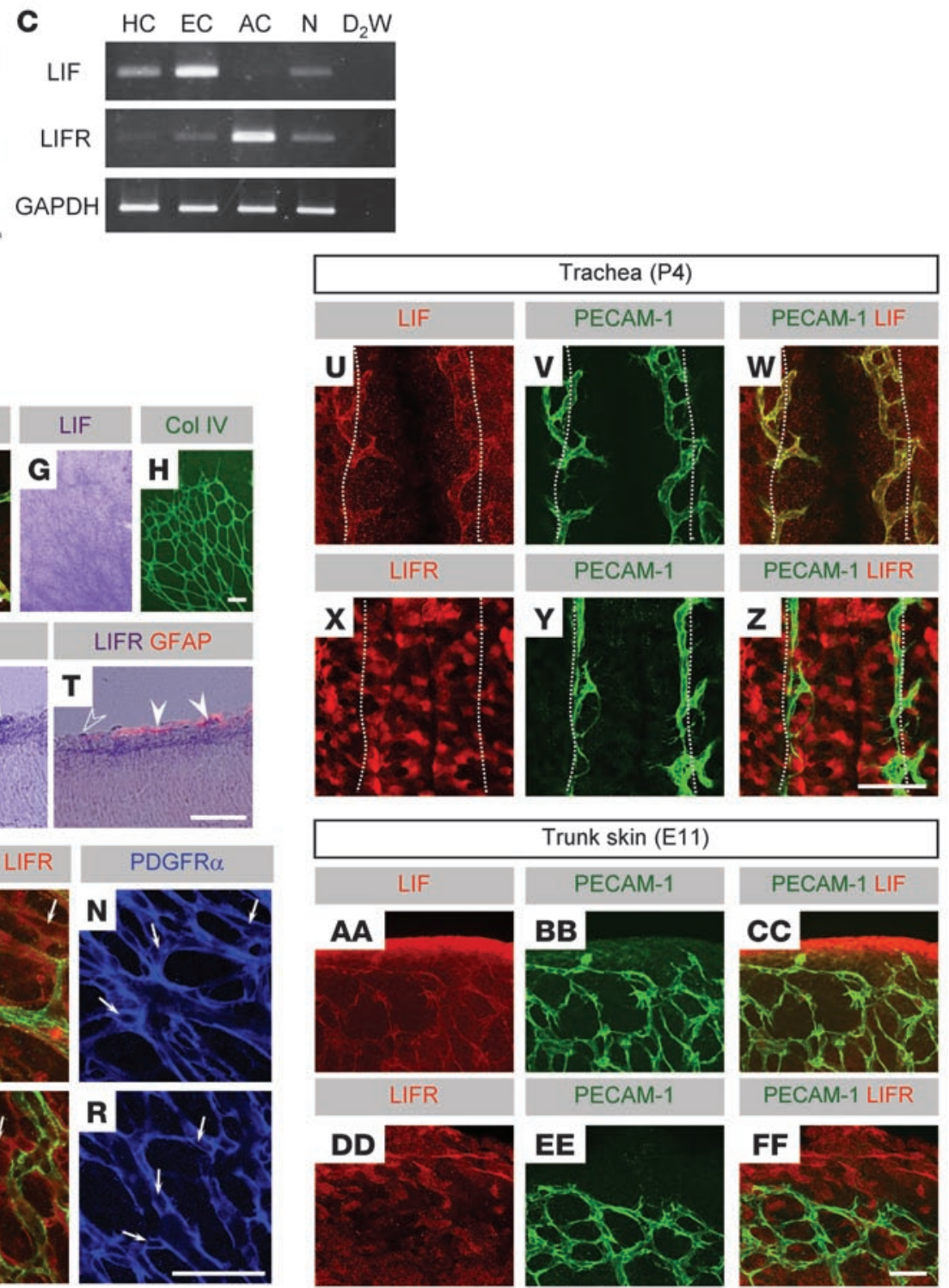

\section{Figure 1}

LIF is predominantly expressed in endothelial cells, and LIFR is expressed in surrounding cells, during vascular development. (A and B) FACS analysis showing 4 sorted fractions of $\mathrm{P} 4$ retina. $\mathrm{HC}$, hematopoietic cells; EC, endothelial cells; AC, astrocytes; N, neurons and other cells. (C) RT-PCR analysis for LIF and LIFR. D 2 W, double-distilled water. (D and E) IHC of LIF on P4 retinas of Lif ${ }^{+/+}$(D) and Lif ${ }^{-1-}(\mathbf{E})$ mice. Boxed region in $\mathbf{D}$ is shown at higher magnification in F. (F) Confocal images scanned for double labeling of LIF and PECAM-1. (G and $\mathbf{H})$ ISH for LIF combined with IHC for collagen IV. LIF was expressed in endothelial cells. (I and J) IHC of LIFR and IgG isotype control staining on P4 retinas. Boxed regions in I are shown at higher magnification in $\mathbf{K}$ and $\mathbf{O}$ as indicated. (S and $\mathbf{T}$ ) ISH on a retinal section at P4 for LIFR combined with IHC for GFAP. LIFR was predominantly expressed in GFAP+ (filled arrowheads) and GFAP- astrocytes (open arrowheads). (K-R) Confocal images scanned for triple labeling of LIFR, PECAM-1, and PDGFR $\alpha$. LIFR was abundantly expressed in astrocytes (arrows) and moderately in neurons. (U-Z) Double IHC for LIF or LIFR and PECAM-1 on P4 tracheal mucosa. Dotted lines flank a cartilaginous ring area. (AA-FF) Double IHC for LIF or LIFR and PECAM-1 on back skin at E11. Scale bars: $50 \mu \mathrm{m}$.

observed outside the retina in both postnatal and embryonic tissues. In the developing cartilaginous ring area of tracheal mucosa (17) at P4, LIF was expressed in the endothelium (Figure 1, U-W), and LIFR was expressed in mucosal epithelial cells (Figure 1, X-Z). In trunk skin at E11, LIF was expressed in the endothelium (Figure 1, AA-CC), and LIFR was expressed in epidermal keratinocytes and dermal cells, presumably dermal fibroblasts (Figure 1, DD-FF). Furthermore, we sought to determine what stimuli upregulate LIF expression in endothelial cells using a human endothelial cell line, HUVECs. VEGF and hypoxia did not induce significant changes in LIF expression in HUVECs (Supplemental Figure 1, A and B; supplemental material available online with this article; doi:10.1172/JCI34882DS1). However, high-glucose stimuli and confluence of cultured cells significantly upregulated LIF expression (Supplemental Figure 1, C and D), although the changes were moderate and HUVECs without stimuli or in sparse 

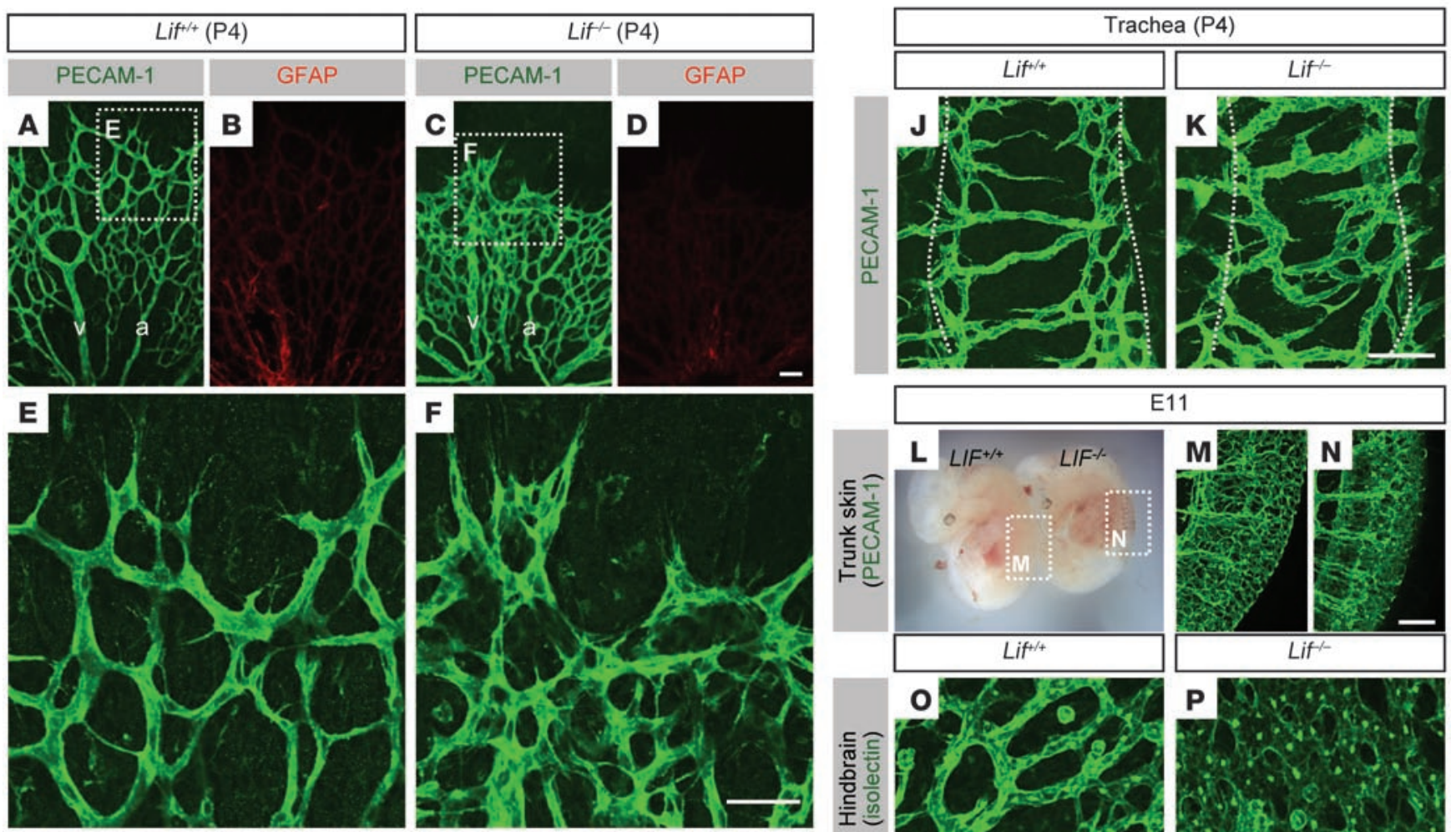

E11
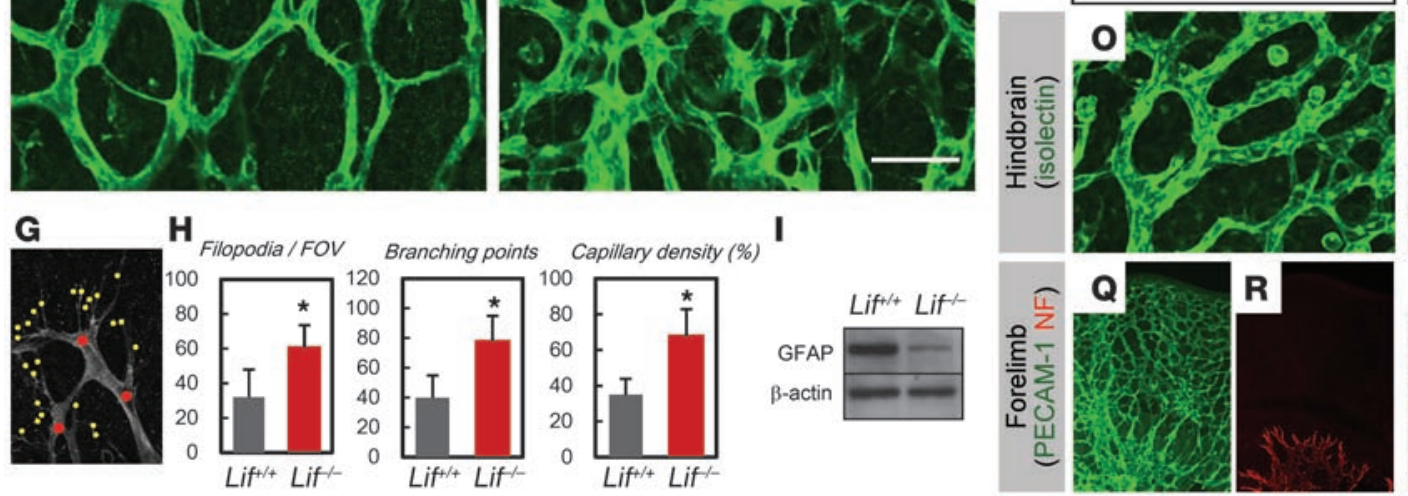

\section{I}
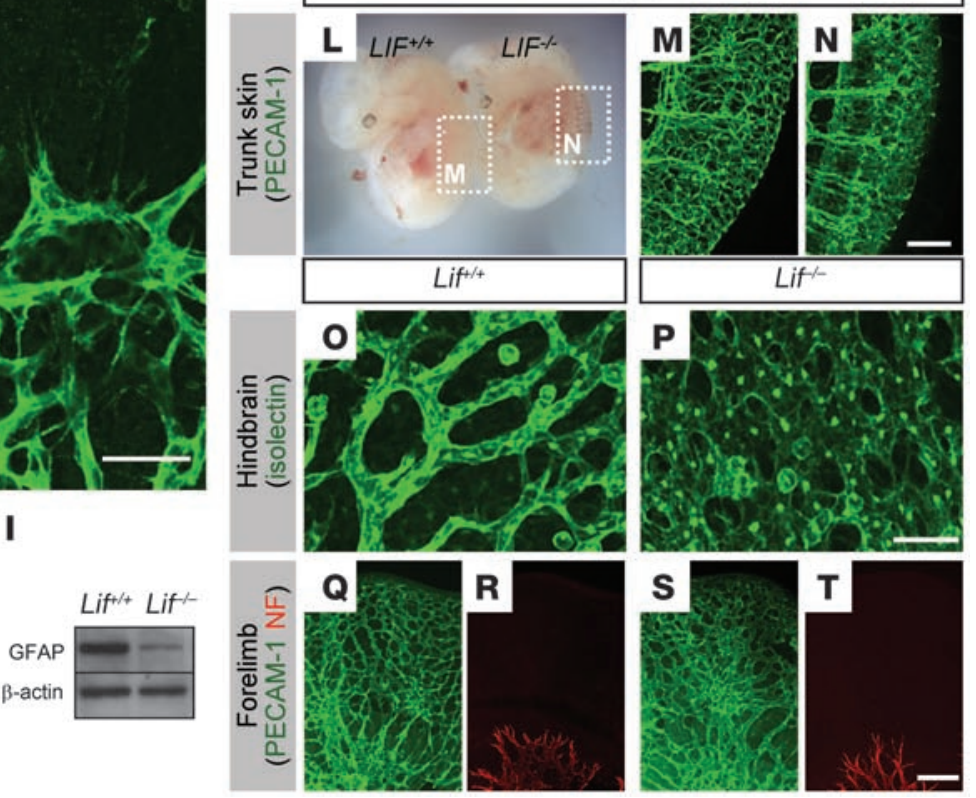

Figure 2

Increased microvessel density and sustained tip cell activity in Lif ${ }^{-1-}$ mice. (A-D) Double IHC of PECAM-1 and GFAP in the P4 retinas of $\mathrm{Lif}^{+/+}$and $\mathrm{Lif}^{-/}$mice. Note the increased microvessel density in contrast to almost normally formed arteries (a) and veins (V). (E and $\mathbf{F}$ ) Higher-magnification images of the boxed regions in $\mathbf{A}$ and $\mathbf{C}$, respectively. Note the tip cells piled up in the Lif ${ }^{-1}$ retina. ( $\mathbf{G}$ and $\left.\mathbf{H}\right) \mathbf{Q u a n t i -}$ fication of the number of filopodia, number of branching points, and capillary density by counting as shown in $\mathbf{G}$. Data are mean numbers from 8 random FOV around the sprouting edge per retina $(n=7)$. (I) Western blotting of GFAP proteins in Lif ${ }^{+/+}$and Lif/- retinas at P4. $(\mathbf{J}$ and K) IHC of PECAM-1 in P4 tracheas. Dotted lines flank a cartilaginous ring area. (L) Appearance of $\mathrm{Lif}^{+/+}$and $\mathrm{Lif}^{/-}$mice at E11. (M and N) Three-dimensional projections for thin-sliced whole-mount E11 embryos stained with PECAM-1 from the indicated boxed regions in L. Note the increased microvessel density, although major trunk vessels and intersomitic vessels were formed normally in $\mathrm{Lif}^{\prime-}$ mice. $(\mathbf{O}$ and P) Isolectin B4 staining for E11 hindbrains. (Q-T) Double IHC of PECAM-1 ( $\mathbf{Q}$ and $\mathbf{S}$ ) and neurofilaments (NF; R and T) in the E12 embryos. Scale bars: $50 \mu \mathrm{m} .{ }^{\star} P<0.03$ versus $\mathrm{Lif}^{+/+}$.

culture also stably expressed LIF. The influence of cell density on LIF expression was examined in vivo via an oxygen-induced retinopathy (OIR) model (18); OIR is characterized by high-density endothelial cell clusters known as neovascular tufts (NVTs). Abundant LIF expression was detected in NVTs, although the remaining normal endothelium also expressed LIF (Supplemental Figure 1, E and F). Expression of LIF even in endothelial tip cells (Figure 1F) suggests that cell density is not the only determinant of LIF expression in endothelial cells and that LIF is constantly expressed in endothelial cells.

Lif ${ }^{-1}$ mice show increased microvessel density accompanied by sustained tip cell activity. To examine the detailed function of LIF in vascular development, we examined retinal angiogenesis in Lif $^{/-}$mice as a primary focus of our study. Retinas of Lifmice showed significantly increased endothelial filopodia and branching points as well as increased capillary density, although major arteries and veins were formed normally (Figure 2, A, C, and $\mathrm{E}-\mathrm{H})$. Lif ${ }^{/-}$mice also showed decreased astrocytic GFAP expression (Figure 2, B, D, and I). In addition, Lif ${ }^{\prime-}$ mice showed increased filopodia and branching in $\mathrm{P} 4$ trachea (Figure 2, J and $\mathrm{K}$ ) and increased microvessel density in trunk skin (Figure 2, $\mathrm{L}-\mathrm{N}$ ) at E11, although major trunk vessels and intersomitic vessels were not affected. Lif ${ }^{\wedge-}$ mice also showed densely condensed capillary networks in the hindbrain plexus (Figure 2, $\mathrm{O}$ and $\mathrm{P}$ ) and forelimbs (Figure 2, Q-T), although nervous development was not affected (Figure 2, R and T). 

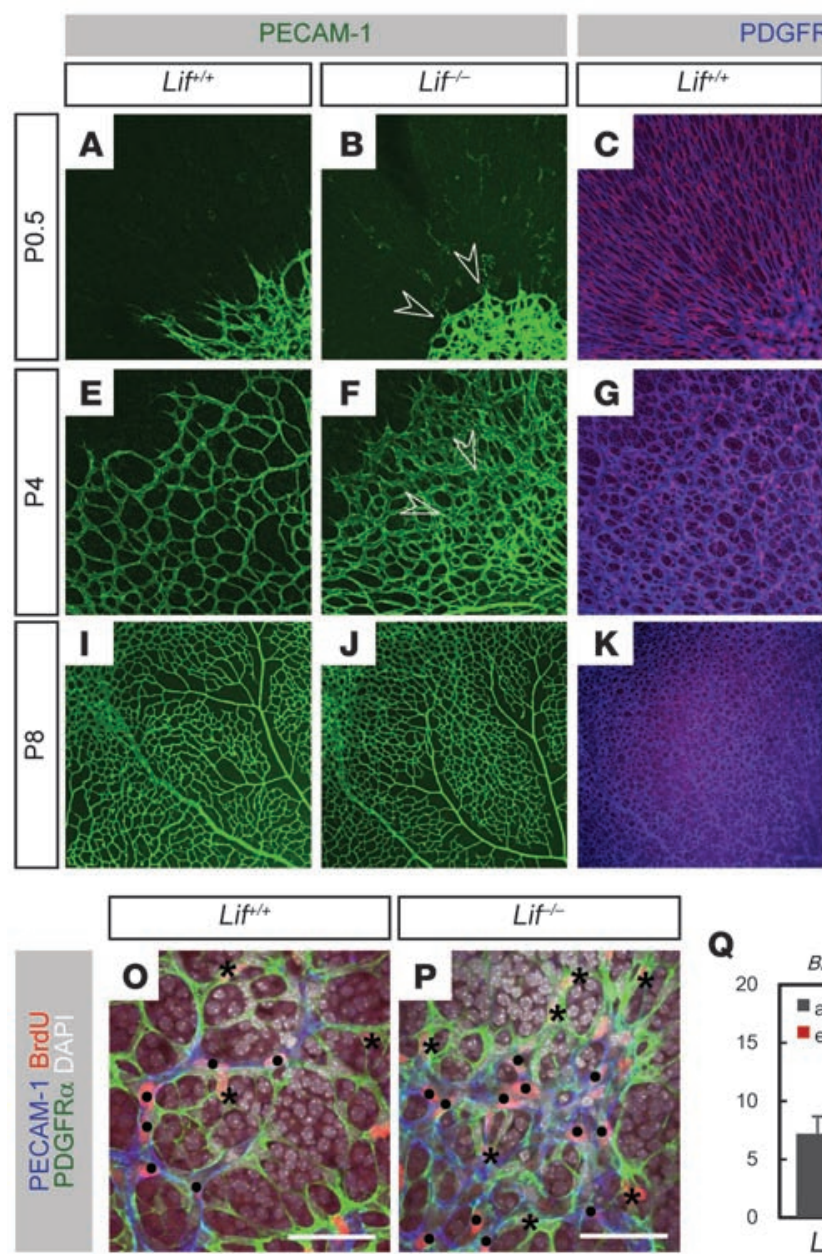
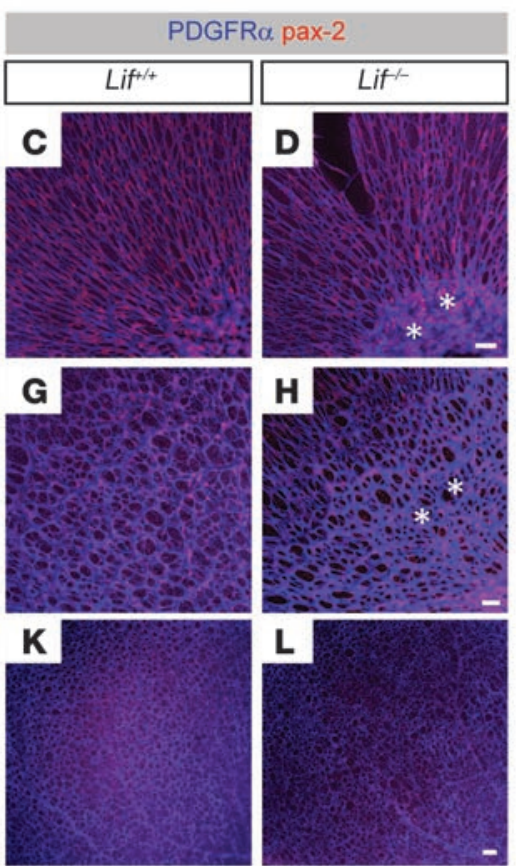

Q

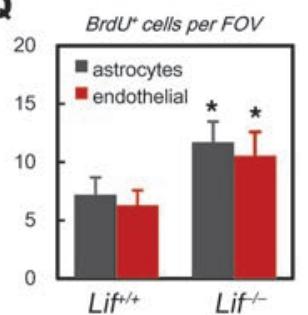

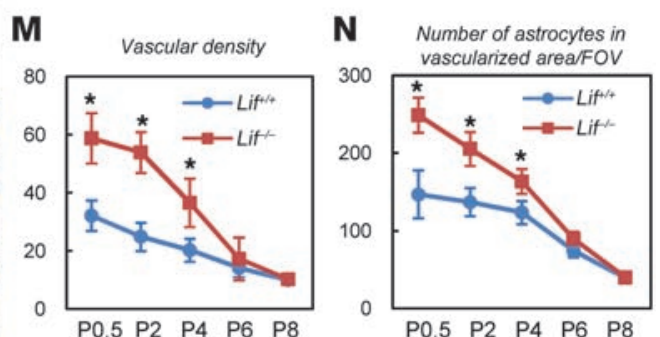
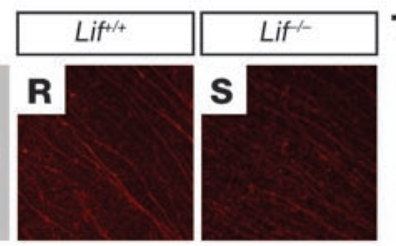

T Relative pdgfa
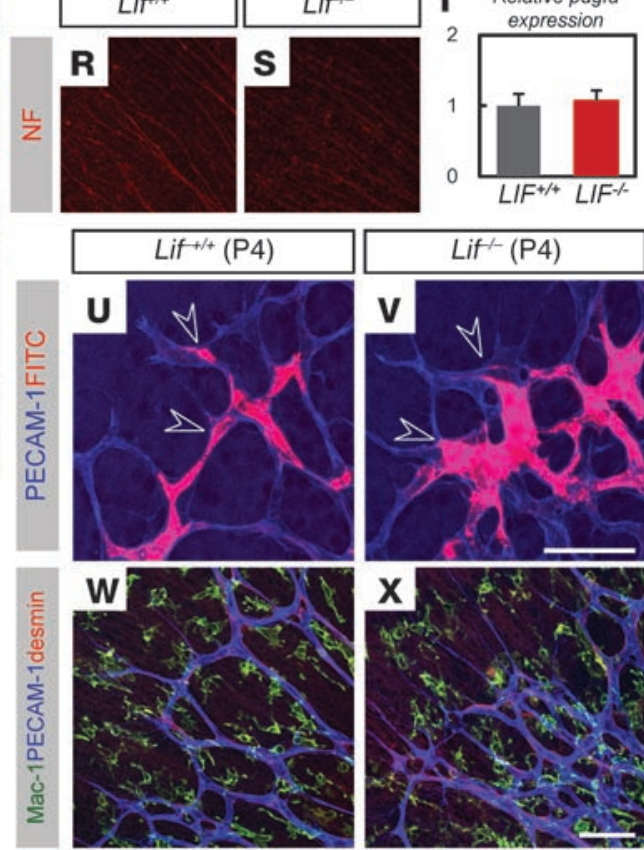

\section{Figure 3}

Lif $^{\prime-}$ mice show increased proliferation in endothelial cells and astrocytes. (A-L) Triple IHC for PECAM-1, PDGFR $\alpha$, and pax-2. Note the densely condensed astrocyte network (asterisks) in the hypervascularized area (arrowheads) of Lif-- mice at P0.5 and P4. (M and N) Transition of vascular density (M) or the number of astrocytes identified as pax-2+ nuclei in vascularized area (N). Data are means of 4-8 random FOV in the vascularized area per retina $(n=5)$. (O and P) BrdU incorporation assay combined with triple IHC for PECAM-1, PDGFR $\alpha$, and DAPI in P4 retinas. Note the BrdU+ astrocytes (asterisks) and endothelial cells (black dots) in the Lif $^{-/}$retina. (Q) Quantification of BrdU+ astrocytes and endothelial cells. Data are means from 8 random FOV around the sprouting edge per retina $(n=7)$. (R and $\mathbf{S}) \mathrm{IHC}$ for neurofilament on P4 retinas. (T) Quantitative PCR of pdgfa for isolated RNA from P4 retinas $(n=5)$. (U and $\mathbf{V})$ Confocal images of FITC labeled with PECAM-1 in the retinas perfused with FITC-dextran. Dextran was perfused into the stalk cells (arrowheads) in both Lif ${ }^{+/+}$and $\mathrm{Lif}^{/-}$mice. (W and X) Triple IHC for PECAM-1, Mac-1, and desmin in P4 retinas. Scale bars: $50 \mu \mathrm{m}$. ${ }^{\star} P<0.03$ versus $\mathrm{Lif}^{+/+}$.

Lif ${ }^{\prime-}$ mice show increased proliferation in endothelial cells and astrocytes. The expression patterns of LIF and LIFR and the decreased GFAP level in retinas of Lif $^{-/}$mice led us to examine the time course profiles of endothelial and astrocyte status. Overview images of pax-2 labeling at various stages with endothelial labeling showed a larger and densely condensed astrocyte network in the hypervascularized area of iff $^{/-}$mice compared with that of wild-type mice (Figure 3, A-L). However, the overgrowth of both endothelium and astrocytes in Lif $^{/-}$mice gradually approached wild-type levels and was not significant at P6-P8 (Figure 3, M and $\mathrm{N}$ ). To assess the proliferation of retinal cells, we performed $\mathrm{BrdU}$ incorporation in retinas of $\mathrm{P} 4 \mathrm{Lif}^{/-}$mice and found the number of proliferating endothelial cells and astrocytes both significantly increased compared with the retinas of wild-type littermates (Figure 3, O-Q). Because PDGF-A secreted from the neural cell body $(5,19)$ functions as a mitogen for retinal astrocytes, we analyzed the status of neurons and PDGF-A expression in if $^{\prime-}$ mice. Neurons did not show any detectable alteration in the Lif ${ }^{\prime-}$ retinas (Figure 3, R and S). In addition, there was no significant difference in quantitative RT-PCR analysis for PDGF-A (Figure 3T). Because astrocyte maturation is oxygen dependent (3) and poorly perfused vessels are expected to fail to promote astrocyte maturation, we tested FITC dextran perfusion and found that retinas of $\mathrm{Lif}^{-1}$ mice were adequately perfused (Figure 3, U and V). Pericytes (20) and microglia, which are relevant to endothelial function, did not show any changes in Lif/- mice (Figure 3, W and X).

Lif $^{\prime-}$ - retinas show increased VEGF in their vascularized area. To examine the proangiogenic state of astrocytes in the retinas of

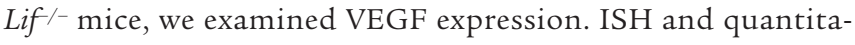




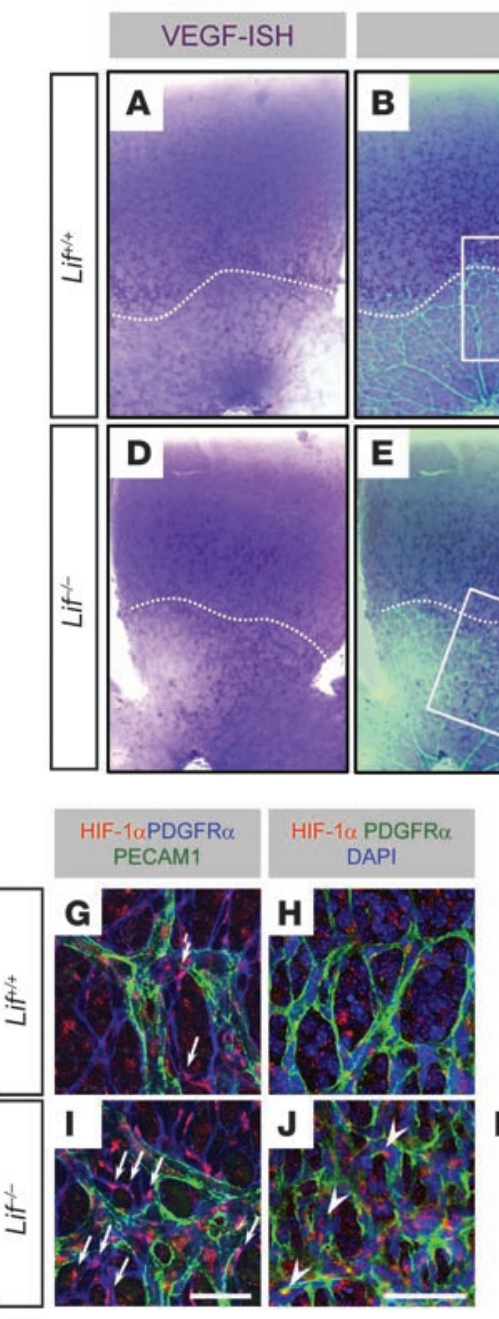

\section{VEGF-ISH Col IV}

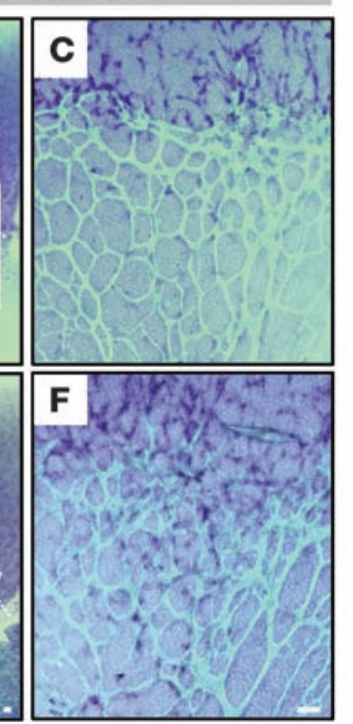

K

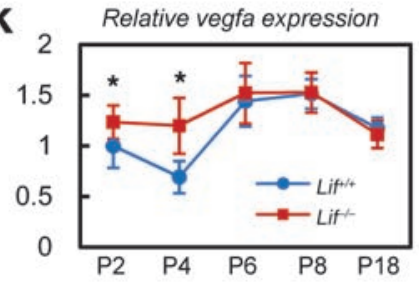

$\mathbf{L}$

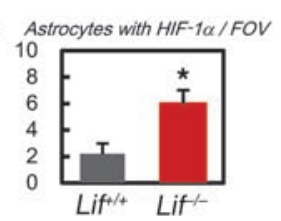

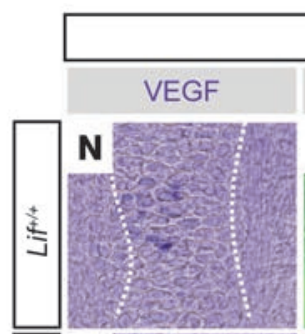
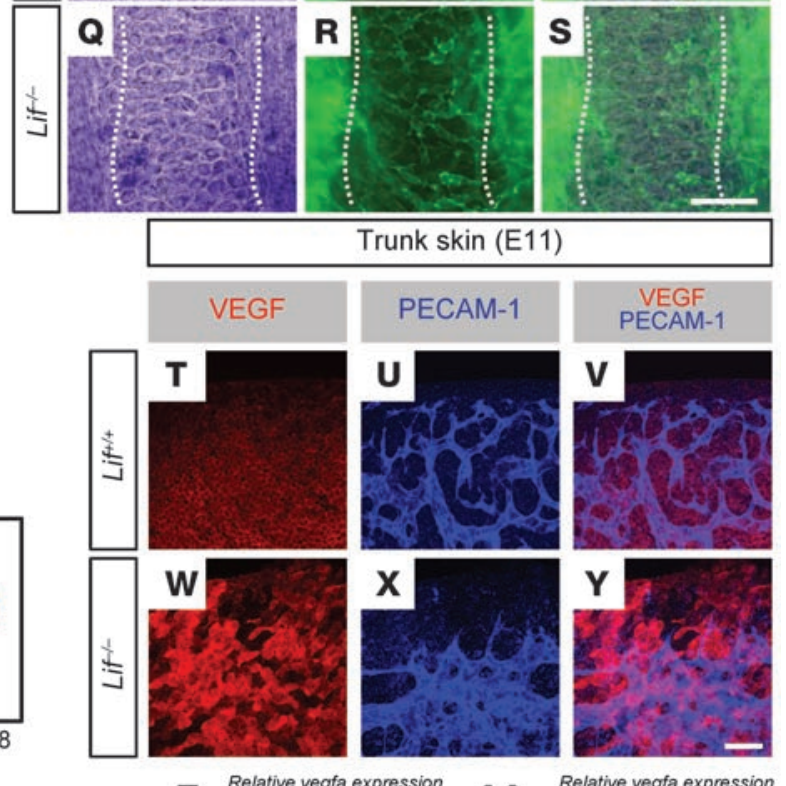

$\mathbf{M}_{\text {Relative hif-1 } 1 \text { expression }}$

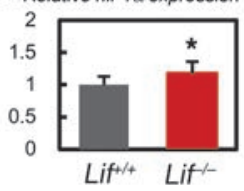

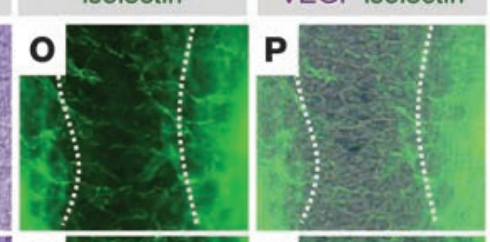
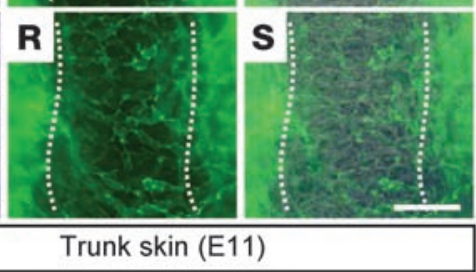

\section{VEGF isolectin}

Relative vegfa expression

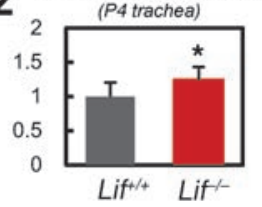

AA

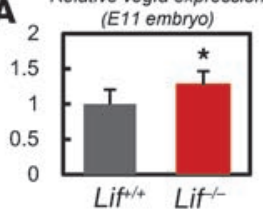

\section{Figure 4}

Increased VEGF expression in the vascularized area of Lif $^{-1}$ retina. (A-F) ISH for VEGF combined with IHC for collagen IV. Note the increase of VEGF-expressing cells in Lif ${ }^{-/-}$mice in the vascularized area. Boxed regions in $\mathbf{B}$ and $\mathbf{E}$ are shown at higher magnification in $\mathbf{C}$ and $\mathbf{F}$ as indicated. Dotted lines in A, B, D, and $\mathbf{E}$ indicate the sprouting edges. (G-J) Triple IHC for PDGFR $\alpha$, PECAM-1, and HIF-1 $\alpha$ (G and I) or for PDGFR $\alpha, \mathrm{HIF}-1 \alpha$, and DAPI (H and $\mathbf{J})$ in P4 retinas of $\mathrm{Lif}^{+/+}$and $\mathrm{Lif}^{/-}$mice. Note the increased number of astrocytes with HIF-1 $\alpha$ (arrows) and nuclear translocated HIF-1 $\alpha$ proteins (arrowheads) in the vascularized area of Lif/- retinas. (K) Quantitative RT-PCR of vegfa with isolated RNA from various retinal stages ( $n=5$ per group). (L) Quantification of astrocytes with HIF-1 $\alpha$ in the vascularized area (average numbers from 8 random FOV in the vascularized area per retina; $n=6$ ). (M) Quantitative PCR of $h i f-1 \alpha$ for isolated RNA from P4 retina $(n=5)$. (N-S) ISH for VEGF combined with isolectin staining in P4 trachea of $\mathrm{Lif}^{+/+}$and $\mathrm{Lif}^{/-}$mice. Note the increase in VEGF-expressing

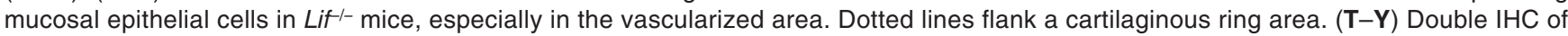
PECAM-1 and VEGF on E11 embryos. (Z and AA) Quantitative PCR of vegfa for isolated RNA from P4 trachea (Z) or E11 embryos (AA) ( $n=5$ per group). Scale bars: $50 \mu \mathrm{m} .{ }^{*} P<0.03$ versus $\mathrm{Lif}^{+/+}$.

tive RT-PCR for VEGF revealed increased VEGF expression at P4 in Lif ${ }^{\prime-}$ mice (Figure 4, A-F and K). Upregulation of VEGF in Lif $^{-1}$ retinas was detected in the vascularized area behind the sprouting edge (Figure 4, E and F). Upregulated VEGF approached wild-type levels in a manner synchronous with the cessation of astrocytic overgrowth at P6-P8 (Figure 3N). Furthermore, because vegf is widely known as a HIF-1 $\alpha$ target gene (21), we examined astrocytic HIF-1 $\alpha$ expression in Lif $^{/-}$- retinas and found significantly increased numbers of astrocytes with HIF- $1 \alpha$ activation and nuclear translocation in the vascularized area (Figure 4, G-J, L, and M). Considering the inverse correlation between VEGF and GFAP expression levels (3) and a previous report showing that LIF induces GFAP expression in cultured astrocytes of the rat optic nerve (22), we checked the in situ VEGF expression in G $\mathrm{fap}^{-/-}$retinas. The results showed that astrocyte expression of VEGF was comparable in wild-type and $\mathrm{Gfap}^{-/-}$retinas (Supplemental Figure 2, A-E). Lif ${ }^{\prime-}$ mice showed normal vasculature with a decrease of GFAP expression at P8 and P18 (Supplemental Figure 3, A-H), while their expression of VEGF was comparable with that of wild-type littermates (Figure 4K). Moreover, although wild-type mice showed VEGF expression only in the avascular central area in $\mathrm{P} 4$ trachea, Lif $^{\prime-}$ mice upregulated VEGF in the mucosal epithelium of the vascularized area (Figure 4, N-S and Z). Epidermal cells and 

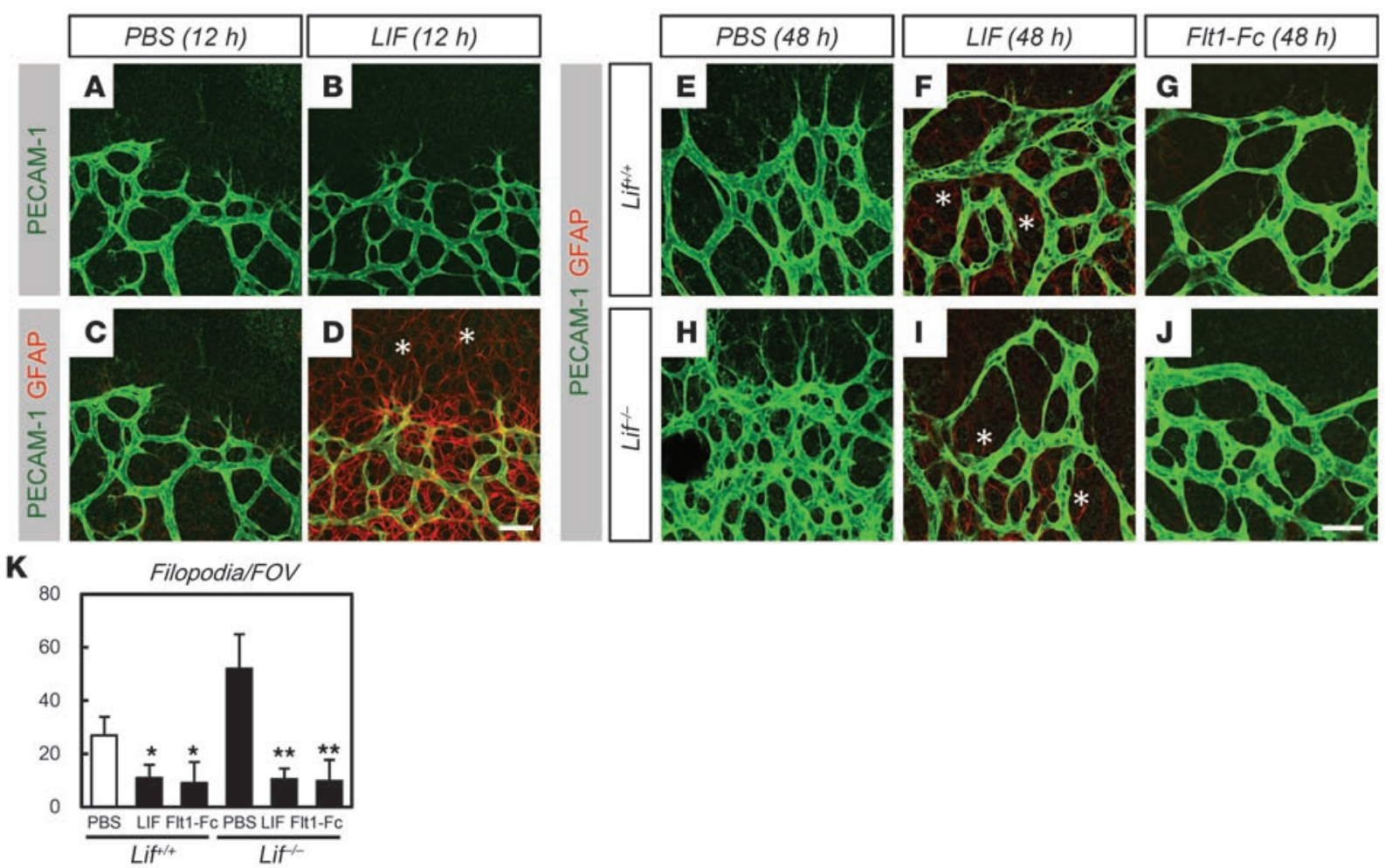

\section{Figure 5}

Increased vascular sprouting in Lif/- mice is inhibited by intraocular injection of LIF or VEGF antagonist. (A-D) Double IHC of PECAM-1 and GFAP on P4 retinas $12 \mathrm{~h}$ after intraocular injection with PBS or LIF. LIF induced upregulation of GFAP ahead of the sprouting edge (asterisks) without inhibiting endothelial sprouting. (E-J) Double IHC of PECAM-1 and GFAP on P5 retinas $48 \mathrm{~h}$ after intraocular injection with PBS, LIF, or Flt1-Fc $(\mathbf{G}$ and $\mathbf{J})$ in the P3 eyes of $\mathrm{Lif}^{+/+}(\mathbf{E}-\mathbf{G})$ or $\mathrm{Lif}^{/-}(\mathbf{H}-\mathbf{J})$ mice. Note that both LIF and Flt1-Fc inhibited endothelial sprouting, while only LIF upregulated GFAP (asterisks). (K) Quantification of the number of filopodia. Data are mean numbers from 8 random FOV in the sprouting edge per retina $(n=6)$. Scale bars: $50 \mu \mathrm{m} .{ }^{*} P<0.03$ versus PBS-injected if $^{+++} ;{ }^{* *} P<0.03$ versus PBS-injected Lif ${ }^{\prime-}$.

dermal fibroblasts in Lif $^{/-}$mice at E11 also showed upregulated VEGF (Figure 4, T-Y and AA).

Increased vascular sprouting in Lif ${ }^{\prime-}$ mice is inbibited by intraocular injection of LIF or VEGF antagonists. To confirm that astrocytes, but not endothelial cells, represent the primary target of LIF in retinal angiogenesis, we examined the short-term effects (i.e., after $12 \mathrm{~h}$ ) of intraocularly injected LIF in wild-type mice. LIF induced maturation of astrocytes even ahead of the sprouting edge without inhibiting endothelial sprouting (Figure 5, A-D). As a long-term effect of LIF injection (i.e., after $48 \mathrm{~h}$ ), LIF significantly inhibited endothelial sprouting, although the upregulation of GFAP beyond the sprouting edge was reversed (Figure 5, E and F) and the entire vascularity of the retina was not affected. This vasoinhibitory effect of LIF injection was also detected when LIF was injected into the eyes of Lif/- mice (Figure 5, H and I). To confirm that the upregulation of VEGF in Lif $^{\prime-}$ mice is attributable to their hypervascular phenotype, we injected a potent VEGF inhibitor, Flt1-Fc chimeric proteins (4), into the eyes of $\mathrm{Lif}^{/-}$mice. As a result, excessive angiogenic sprouting in Lif ${ }^{\prime-}$ mice was significantly inhibited even to a level below that of wild-type retinas injected with vehicle only (Figure 5, E, J, and K).

LIF antagonizes the bypoxia-induced proangiogenic state of astrocytes via the JAK/STAT pathway in vitro. The above results indicated that LIF modulates the oxygen-dependent proangiogenic state of astrocytes. Therefore, we examined whether such an effect occurs in in vitro experiments. We added exogenous LIF to a previously described (3) retinal culture system (Figure 6, A-T). Consistent with this previous report, GFAP expression (Figure 6, A and C) was suppressed, while astrocyte proliferation (Figure 6, F and H) and VEGF expression (Figure 6, K and M) increased under hypoxic conditions. When the hypoxic culture was supplemented with LIF, these features of the astrocytic hypoxic response were all inhibited: GFAP was significantly upregulated (Figure 6, C, D, and U), while proliferation (Figure 6, H, I, and V) and VEGF expression (Figure 6, M, N, and W) decreased significantly compared with hypoxic cultures without LIF. Moreover, LIF significantly increased the number of p-STAT3-expressing astrocytes in the hypoxic culture; these are normally suppressed in hypoxia (Figure 6, P-S and X). In a TUNEL assay, LIF treatment of astrocytes in hypoxic culture did not increase apoptosis (Supplemental Figure 4, A and B). Because these results suggested the LIFR-JAK/STAT pathway induces the cessation of the proangiogenic state of hypoxic astrocytes directly or indirectly, we added an inhibitor of JAK2 activity, AG490, into the LIF-supplemented hypoxic culture of astrocytes. AG490 inhibited the antagonistic effects of LIF against hypoxia, upregulation of GFAP, inhibition of proliferation, and expression of VEGF in hypoxic astrocytes (Figure 6, $\mathrm{E}, \mathrm{J}, \mathrm{O}, \mathrm{T}$, and $\mathrm{U}-\mathrm{X}$ ). Vehicle (DMSO) supplementation as a 

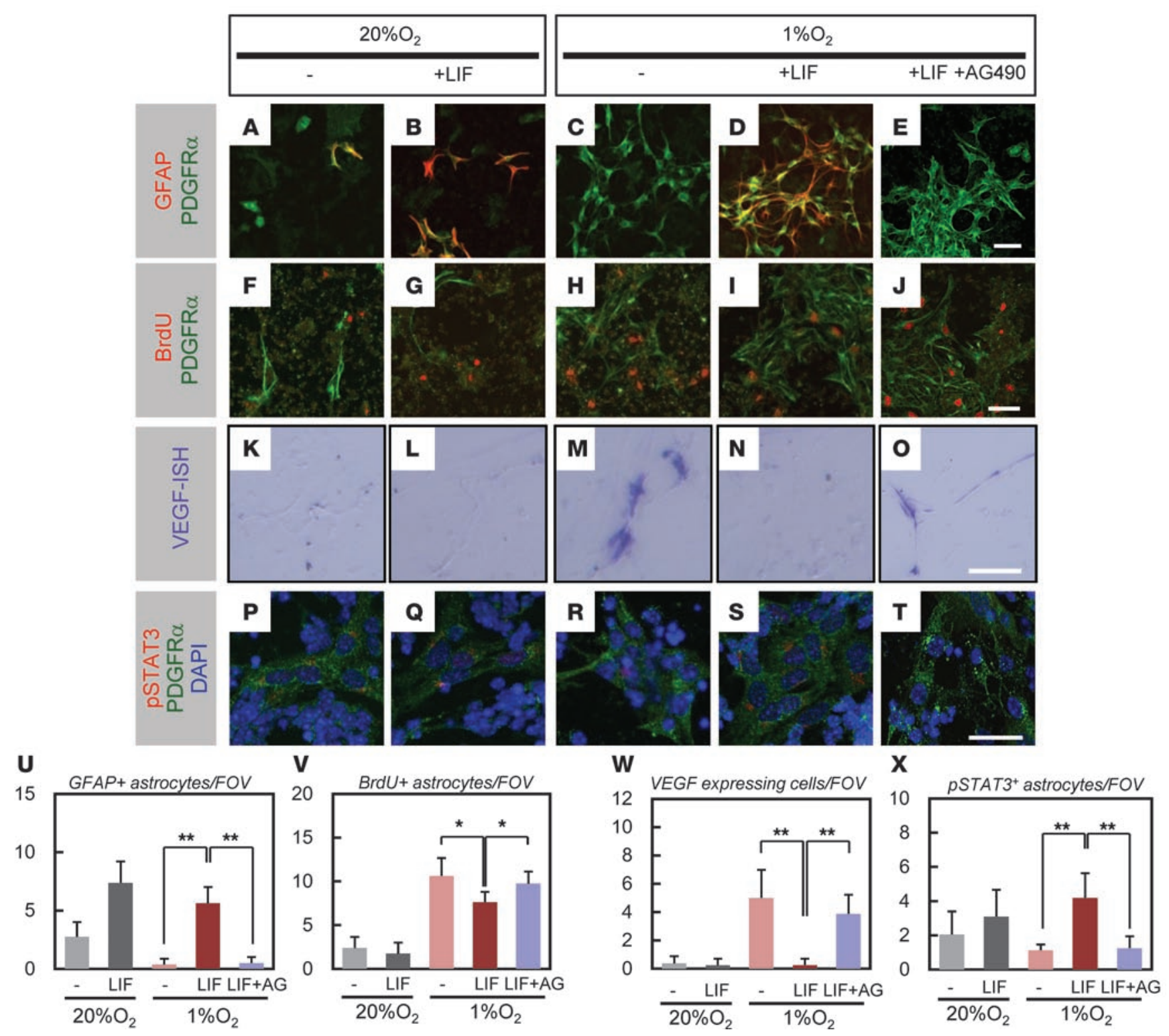

Figure 6

LIF antagonizes the hypoxia-induced proangiogenic state of astrocytes via the JAK/STAT pathway in vitro. (A-T) Representative images of

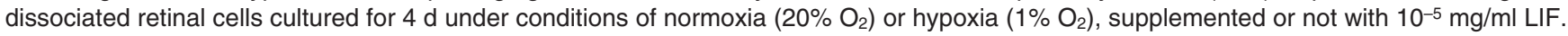
AG490 $(200 \mu \mathrm{M})$ was added in some experiments. (A-E) IHC for PDGFR $\alpha$ and GFAP. (F-J) BrdU incorporation assay combined with IHC for PDGFR $\alpha$. (K-O) ISH for VEGF. (P-T) IHC for PDGFR $\alpha$ and p-STAT3. (U-X) Quantification of the number of GFAP+ astrocytes $(\mathbf{U})$, BrdU ${ }^{+}$ astrocytes $(\mathbf{V})$, VEGF-expressing cells $(\mathbf{W})$, and p-STAT3+ astrocytes $(\mathbf{X})$. Data are means from 8 random FOV per experiment $(n=5)$. Scale bars: $50 \mu \mathrm{m} .{ }^{\star} P<0.03 ;{ }^{\star \star} P<0.01$.

control of AG490 did not show any significant effects in these experiments (data not shown).

LIF deficiency causes resistance against byperoxic insult and increased extraretinal neovascularization in an OIR model. Vascular defects of Lif ${ }^{\prime-}$ mice and their altered VEGF expression were further examined in the OIR model (18). The retinas of Lif $^{\prime-}$ mice showed significant resistance against hyperoxygen insult and significantly increased VEGF expression (Figure 7, A-L). In the revascularization phase, Lif ${ }^{\prime-}$ mice showed significantly increased NVT formation compared with wild-type mice (Figure 7, M-T and W). Severe hemorrhage was seen in Lif/- mice (Figure 7, $\mathrm{U}$ and V); this was presum- ably the result of an insufficient blood-retina barrier, as reflected by decreased GFAP expression (Figure 7, N and P-R). VEGF expression in the retinas of Lif $^{/-}$mice significantly increased compared with wild-type mice during the revascularization phase (Figure 7, X and $\mathrm{Y}$ ). Considering the abundant expression of LIF in NVTs (Supplemental Figure 1, E and F), these results indicate that LIF confers a physiological defense mechanism against retinal pathological angiogenesis. Therefore, we tested the beneficial effect of exogenous LIF administration in the OIR model. LIF injections at P15 significantly ameliorated the severity of the OIR, as evidenced by the decreased avascular area and NVT area at P17 (Figure 8, A-F). 


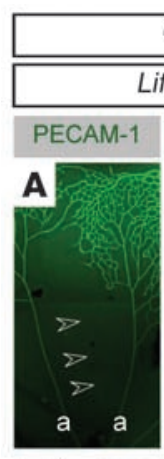

OIR P12 (vaso-obliteration)

\begin{tabular}{llll} 
& \\
\hline
\end{tabular}
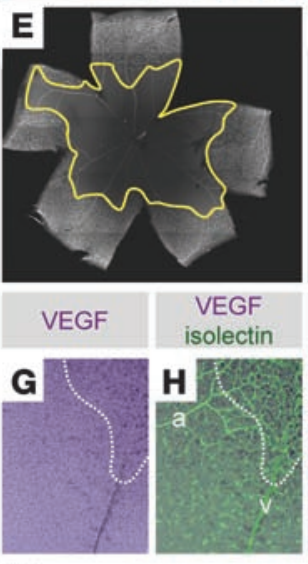

$\mathbf{K}$

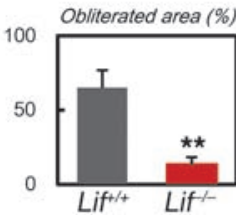

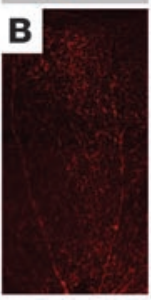
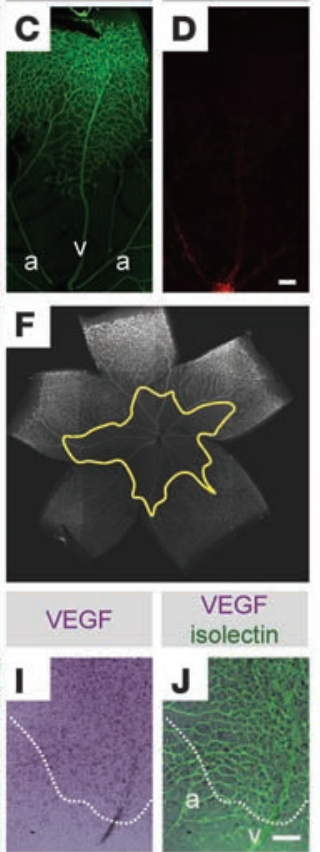

L Relative vegfa expression

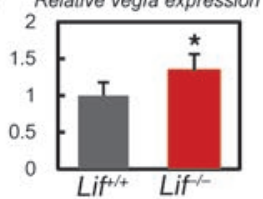

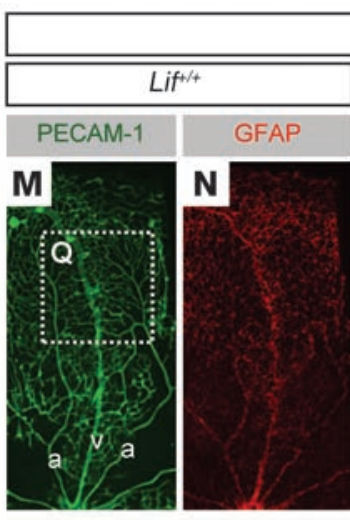

PECAM-1 GFAP

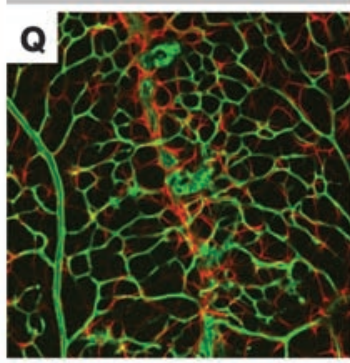

s

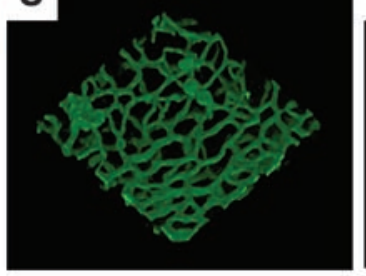

OIR P17 (neovascular tufts)

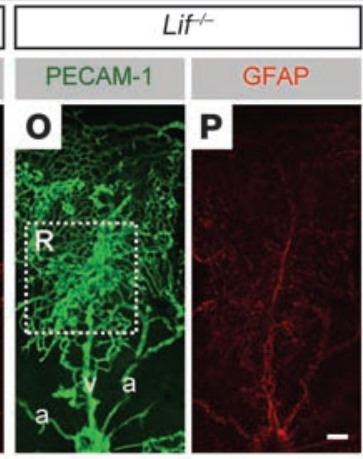

PECAM-1 GFAP

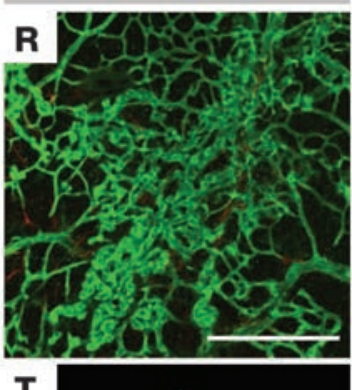

T

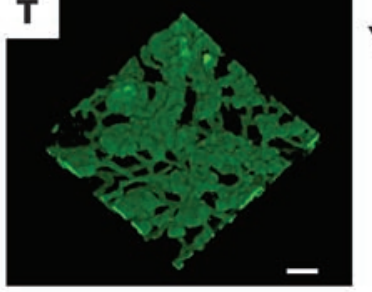

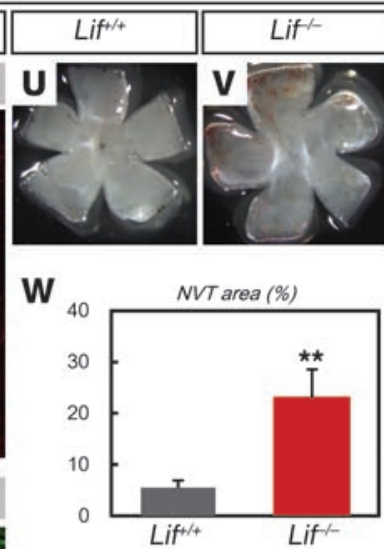

X Relative vegfa expression

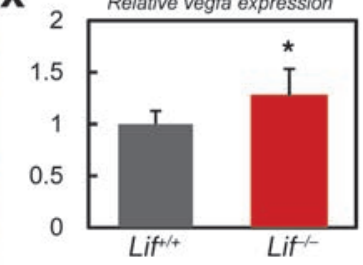

$\mathbf{Y}$

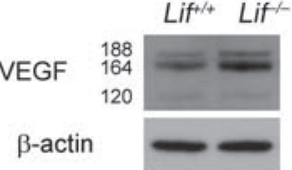

Figure 7

LIF deficiency causes resistance against hyperoxic insult and increased extraretinal neovascularization in the OIR model. (A-F) Double IHC of PECAM-1 (green in $\mathbf{A}$ and $\mathbf{C}$; white in $\mathbf{E}$ and $\mathbf{F}$ ) and GFAP (red in $\mathbf{B}$ and $\mathbf{D}$ ) in P12 retinas after 3 days of hyperoxygen insult. Note the obliterated vein typically observed in $\mathrm{Lif}^{+/+}$mice (open arrowheads), which was preserved in $\mathrm{Lif}^{-/}$mice. Also note the entirely decreased area of vaso-obliteration (yellow outlines in $\mathbf{E}$ and $\mathbf{F}$ ) in Lif-/- mice. (G-J) ISH for VEGF combined with isolectin staining in P12 retinas in the OIR model. Note the increased VEGF-expressing cells in Lif ${ }^{-1}$ mice were detected in the area resistant against hyperoxygen insult (above dotted lines). (K) Quantification of the vaso-obliterated area. Data represent quantification of the entire retina $(n=6)$. (L) Quantitative RT-PCR of vegfa for RNA isolated from P12 retina in the OIR model $(n=5)$. (M-P) Double IHC of PECAM-1 and GFAP in P17 retinas in the OIR model. ( $\mathbf{Q}$ and $\mathbf{R}$ ) Higher-magnification images of the boxed regions in $\mathbf{M}$ and $\mathbf{O}$, respectively. (S and $\mathbf{T})$ Three-dimensional confocal images of IHC for PECAM-1 in the NVT area. (U and V) Bright-field images of P17 retinas of Lift++ and Lif ${ }^{/-}$mice in the OIR model. Note the severe bleeding in the NVT area of Lif ${ }^{-1}$ retina. (W) Quantification of the NVT area. Data represent quantification of the entire retina $(n=7)$. (X) Quantitative RT-PCR of vegfa for RNA isolated from P17 retina in the OIR model $(n=5)$. (Y) Western blotting of VEGF proteins from P17 retina in the OIR model. Numbers indicate VEGF ${ }_{120}, V_{E G F}{ }_{164}$, and VEGF 188 isoforms, respectively. a, artery; $v$, vein. Scale bars: $50 \mu \mathrm{m} .{ }^{*} P<0.03,{ }^{*} P<0.01$ versus $\mathrm{Lif}^{+/+}$.

\section{Discussion}

In this study, we found that LIF, which is predominantly secreted by endothelial cells, is essential for proper vascular network formation in the proposed model: LIF inhibits VEGF expression and proliferation in astrocytes by inducing astrocytes to differentiate and express GFAP, thus counteracting their response to hypoxia (Figure 9). Our results in tissues outside the retina strongly suggest that similar mechanisms underlie establishment of the capillary network in various tissues. This endothelial negative feedback signal, which depends on LIF, globally plays an important role in the reciprocal feedback mechanism that ensures adequate tissue vascularization.
Concerning the correlation between oxygen concentration and LIF, the VEGF expression pattern in the retinas of Lif $^{\prime-}$ mice (Figure 4, E and F) suggests that regulatory mechanisms of VEGF expression in retina are spatially separated: VEGF expression level is dependent on oxygen concentration in the avascular area. On the other hand, LIF confers regulation of VEGF in the vascularized area cooperatively with oxygen. The unique response of $\mathrm{Lif}^{-1}$ mice to the manipulated oxygen level in the OIR model illustrated that VEGF was cooperatively regulated by oxygen and endothelium-derived LIF. LIF deficiency caused resistance against hyperoxic insult, but increased NVT formation. This feature is paradoxical in view of wild-type or other 

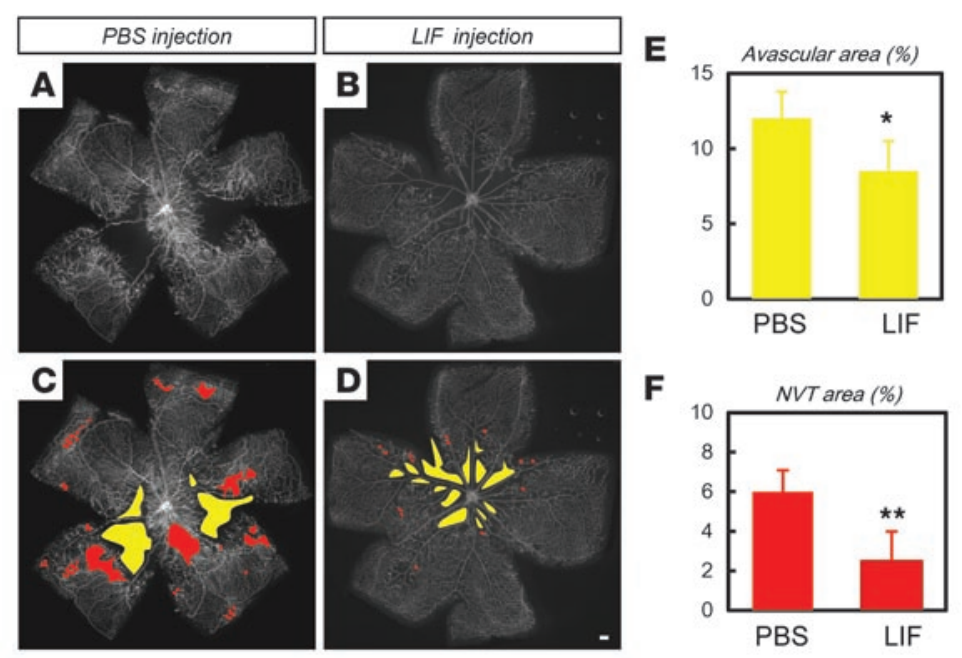

Figure 8

Exogenous LIF ameliorates pathological angiogenesis. (A-D) IHC of PECAM-1 in the P17 retinas of intravitreously injected with PBS or LIF at P15 in the OIR model. Quantification methods are illustrated in $\mathbf{C}$ and $\mathbf{D}$. Note the decreased avascular area (yellow) and NVT area (red) in LIF-injected mice. (E and F) Quantification of the avascular area $(\mathbf{E})$ and NVT area $(\mathbf{F})$. Data represent quantification of the entire retina $(n=6)$. Scale bars: $50 \mu \mathrm{m} .{ }^{*} P<0.03,{ }^{* \star} P<0.01$ versus PBS.

genetically manipulated mice that show alteration in their response to the OIR model: decreased obliterated area is usually expected to lead to mild NVT formation (23). In the gain-offunction study (Figure 5, A-D), LIF affected astrocytes in the vascularized area. Although one would expect a drastic response over the entire retina and even vessel regression after LIF injection, the entire vascularity of the retina was not affected. It is likely that VEGF expression could be maintained in astrocytes after they were exposed to exogenous LIF.

The astrocytic phenotypes we observed in retinas of Lif/- mice were similar to those of transgenic mice overexpressing PDGF-A in retinal neurons (5) or of GFAP-PDGF-A mice (4). Both of these mice $(4,5)$ show a large increase in the number of retinal astrocytes and consequent overgrowth of the retinal vasculature. Nevertheless, a stable steady state follows, and the overall retinal architecture is preserved. The authors concluded that the cessation of astrocytic proliferation is caused by a negative feedback signal from the endothelial cells that counteracts PDGF-Ainduced proliferation, and oxygen in blood flow was suggested to be one of the candidate factors mediating this counteraction. Considering these findings, the expression of LIFR in astrocytes and neurons suggests the possibility that LIF negatively regulates not only astrocyte growth, but also a neuronal function that leads to proangiogenic events. Therefore, we examined the morphology, cell number, and PDGF-A expression level in retinas of Lif/- mice, but found no detectable abnormalities (Figure 3, R-T). Compensatory activation of gp130 signaling by other members of the IL-6 superfamily, such as ciliary neurotrophic factor and IL-6 (24), might be responsible for the lack of detectable neuronal abnormalities in Lif/- mice.

A previously described inverse correlation between VEGF and GFAP expression levels (3) raised the possibility that upregulated VEGF in Lif/- mice results from decreased GFAP expression. Our finding that VEGF expression was comparable in wild-type and
Gfap ${ }^{-/-}$retinas (Supplemental Figure 2, A-D) demonstrates that decreased GFAP was not the cause of inhibited VEGF in Lif/- mice, but was the consequence of their impairment in astrocytic maturation. This conclusion is supported by the observed normal vascular phenotype and VEGF expression despite the decrease of GFAP expression in Lif//- mice at P8 and P18 (Supplemental Figure 3), which also suggests that they were favored by oxygen or other members of the IL-6 superfamily (24) to compensate for LIF deficiency.

HIF-1 is an oxygen-dependent transcriptional activator whose stability and activity is regulated by various types of posttranslational modification, including hydroxylation, acetylation, and phosphorylation (25). The increased number of astrocytes expressing activated HIF-1 $\alpha$ in Lif $^{\prime-}$ mice (Figure 4, G-J and L) and the inhibitory effects of AG490 against in vitro activity of LIF on hypoxic astrocytes suggest that the LIF/STAT3 pathway affects the stability or activity of HIF-1 $\alpha$ directly or indirectly. Recently, it was shown that hypoxic culture conditions induce the differentiation of mouse ES cells even in the presence of LIF (26). Although this in vitro data differs from our in vivo findings that endothelium-derived LIF induced differentiation of HIF- $1 \alpha$-expressing astrocytes, it is likely that there is some cross-talk at the molecular level between LIF/STAT3 and HIF-1 $\alpha$ signals. A recent study showed activation of mammalian target of rapamycin (mTOR) signaling enhances HIF-1 $\alpha$ transcription (27). Our results that Lif/- mice showed moderately but significantly increased HIF- $1 \alpha$ transcription might suggest a direct or indirect link to mTOR signaling. It will be of interest to clarify the precise mechanisms for the molecular cross-talk and functional diversity at the cellular differentiation level, although it is possible that LIF is more important in shutting down astrocyte proliferation and thus reducing the number of VEGF-expressing cells than in directly altering HIF activity.

Our present findings show that LIF modulates oxygen-dependent VEGF expression and is essential for ensuring proper capillary density. In the future, targeting LIF signaling might be a new strategy for antiangiogenic therapy in human diseases such as diabetic retinopathy and cancer.

\section{Methods}

Mice and hyperoxia exposure. C57BL/6 mice (SLC), Lif/- mice (13), and $\mathrm{Gfap}^{-/}$mice (28) were used in these studies. Both mutant mouse strains have been intermittently backcrossed with the C57BL/6 strain. The Keio University Ethics Committee for Animal and Recombinant DNA Experiments (Tokyo, Japan) reviewed and approved all experiments in this study. For the OIR model, P9 mice with nursing mothers were maintained for 3 days in $80 \%$ oxygen, and then placed back in room air, a modification of the conventional method (18).

Preparation of whole-mount samples of retinas, tracheas, bindbrains, and forelimbs. Enucleated eyes, isolated tracheas, or whole embryos were fixed for $20 \mathrm{~min}$ in 4\% PFA in PBS and then dissected. The obtained tissues were postfixed overnight in $4 \% \mathrm{PFA}$ in PBS and stored in methanol at $-20^{\circ} \mathrm{C}$.

Whole-mount immunostaining and ISH. IHC for whole-mount retinas and other tissues was performed as described previously (4). The primary antibodies used were anti-PECAM-1 (2H8; Chemicon International), antiGFAP (G-A-5, Cy3 conjugated; Sigma-Aldrich), anti-PDGFR $\alpha$ (APA5; 


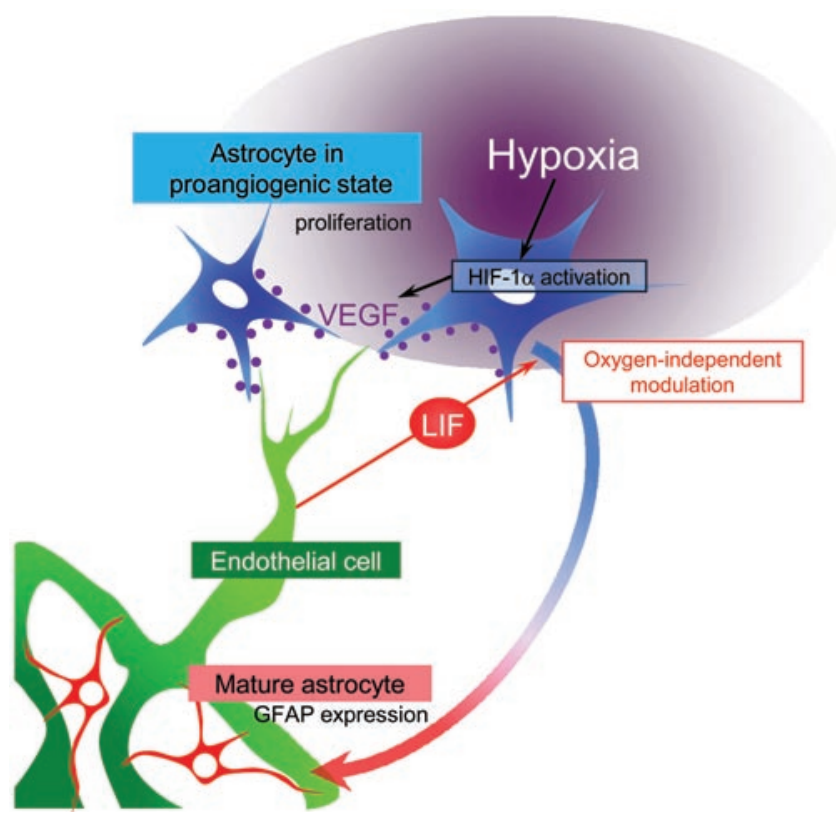

eBioscience), anti-neurofilament (2H3; Developmental Studies Hybridoma Bank), anti-Mac-1 (M1/7; BD Biosciences - Pharmingen), anti-desmin (DAKO), polyclonal anti-type IV collagen (Cosmo Bio), anti-LIFR $\beta$ (Santa Cruz Biotechnology Inc.), anti-pax-2 (Covance), anti-LIF (Santa Cruz Biotechnology Inc.), anti-p-STAT3 (Tyr705; Cell Signaling), and anti-HIF-1 $\alpha$ (Santa Cruz Biotechnology Inc.). The secondary antibodies used were Alexa Fluor 488-conjugated IgGs (Invitrogen) or Cy3/Cy5-conjugated IgGs (Jackson ImmunoResearch Laboratories). For nuclear staining, specimens were treated with DAPI (Invitrogen). For whole-mount ISH, retinas were briefly digested with proteinase $\mathrm{K}$ and hybridized with digoxigenin-labeled antisense RNA probes. When ISH was combined with IHC, IHC was performed after all ISH procedures were completed. For the $\mathrm{BrdU}$ incorporation assay, $100 \mu \mathrm{g} / \mathrm{g}$ body weight of BrdU (BD Biosciences - Pharmingen) dissolved in sterile PBS was injected intraperitoneally $2 \mathrm{~h}$ before sacrifice. Isolated retinas were stained using a BrdU IHC system (Calbiochem) according to the manufacturer's instructions. When BrdU assays were combined with IHC, the application of first antibodies was done simultaneously. For visualization of vascular lumina, FITC-conjugated dextran (Sigma-Aldrich) was injected into the left cardiac ventricle and allowed to circulate for $5 \mathrm{~min}$. After staining, samples were mounted using a Prolong Antifade Kit (Invitrogen).

Western blotting. Western blot analysis was performed as described previously (29). The first antibodies used were anti-VEGF (Santa Cruz Biotechnology Inc.) or anti-GFAP (Sigma-Aldrich). The amount of total protein was examined by reblotting with anti- $\beta$-actin (Sigma-Aldrich).

FACS of dissociated retinal cells. Retinas of $\mathrm{P} 4$ mice were incubated for 30 minutes at $37^{\circ} \mathrm{C}$ in DMEM containing $1 \%$ collagenase D (from Clostridium bistolyticum) before the cells were dissociated by gentle trituration. After immunostaining of harvested cells, cell purification and analysis were performed using FACS Vantage (BD), and cells were analyzed with CELLQuest software version $3.3(\mathrm{BD})$.

RT-PCR analysis. Total RNA was prepared from cells sorted by FACS or from retinal tissues and reverse transcribed using Superscript II (Invitrogen). cDNA samples were then subjected to PCR amplification using the following primers: LIF, 5'-CCCTTACTGCTGCTGGTTC-3' and 5'-TGGACCACCACACTTATGAC-3'; LIFR, 5'-CAGGTTCCATTCTTGTCACC-3' and 5'-CGGTAGGTCTCGTTTGTAAG-3'; GAPDH, 5'-AATCCCATCAC-

\section{Figure 9}

Proposed model of the negative feedback mechanism of LIF against hypoxia-induced VEGF expression in astrocytes. Tissue hypoxia maintains astrocytes in a proangiogenic state, reflected by abundant HIF-1 $\alpha$ activation and VEGF expression and proliferation. On the other hand, LIF secreted from endothelial cells triggers maturation of astrocytes; these mature astrocytes express GFAP, but not VEGF.

CATCTTCCA-3' and 5'-CCAGGGGTCTTACTCCTTG-3'. A quantitative PCR assay was performed on an ABI 7500 Fast Real-Time PCR System using TaqMan Fast Universal PCR master mixture (Applied Biosystems) and TaqMan Gene Expression Assay Mix of vegfa (Mm00437304_m1),pdgfa (Mm01205760_m1) orlif(Hs01055668_m1).Mouse $\beta$-actin(Mm00607939_s1) or human $\beta$-actin (Hs03023880_g1) assay mix served as an endogenous control. Data were analyzed by 7500 Fast System SDS Software version 1.3.1. All experiments were done with 4 replicates.

Cell culture. Culturing of retinal cells was performed as described previously (3). Retinas of P1 neonates were dissociated as described above. Cells were washed and resuspended in DMEM (Sigma-Aldrich) containing 10\% FBS and then plated on poly-D-lysine-coated culture slides at a density of $7.5 \times 10^{5} \mathrm{cells} / \mathrm{cm}^{2}$. The samples were incubated at $37^{\circ} \mathrm{C}$, in $20 \%$ (normoxic) or $1 \%$ (hypoxic) $\mathrm{O}_{2}$. For some experiments, the culture medium was supplemented with LIF (ESGRO; Chemicon) at a concentration of $10^{-5} \mathrm{mg} / \mathrm{ml}$ with or without $200 \mu \mathrm{M}$ of the selective JAK2 inhibitor tyrphostin AG490 (Sigma-Aldrich). After 4 days of culture, cells were fixed with $4 \%$ PFA in PBS for $30 \mathrm{~min}$ and subjected to various analyses. For TUNEL assays, retinas were stained using the ApopTag Fluorescein In situ Apoptosis Detection Kit (Chemicon) according to the manufacturer's instructions. HUVECs were cultured in EGM-2 medium (Cambrex). In VEGF induction, cells were starved in EBM-2 medium without serum or growth factors for $12 \mathrm{~h}$ before applying $10 \mathrm{ng} / \mathrm{ml}$ human VEGF (Peprotech). HUVECs were exposed to $1 \%$ oxygen conditions for hypoxic culture or to $30 \mathrm{mmol} / \mathrm{l}$ glucose for highglucose culture. To obtain sparse or confluent cultures of HUVECs, 1,800 or 42,000 cells $/ \mathrm{cm}^{2}$ were seeded and cultured for $48 \mathrm{~h}$.

Intravitreous injections. Injections into the vitreous body were performed using 33-gauge needles as described previously (4). Sterile PBS (0.5 $\mu \mathrm{l})$ with or without $1 \mathrm{mg} / \mathrm{ml}$ LIF (ESGRO; Chemicon) or Flt1-Fc chimera proteins (R\&D Systems) were injected at P3, P4, or P15 in the OIR model.

Confocal microscopy and quantification. Fluorescence images were obtained using a confocal laser scanning microscope (FV1000; Olympus) at room temperature. Quantification of the cells or substances of interest was usually done in 8 random $200 \times 200 \mu \mathrm{m}$ fields of view (FOV) per retina. To construct 3-dimensional projections, multiple slices horizontally imaged from the same FOV were integrated using FV10-ASW Viewer (Olympus). For constructing images for quadruple IHC, triple-colored images were overlaid with DAPI using Adobe Photoshop CS2.

Statistics. All results are expressed as mean $\pm \mathrm{SD}$. Comparisons between the mean variables of 2 groups were made by 2 -tailed Student's $t$ test. $P$ values less than 0.03 were considered to be statistically significant.

\section{Acknowledgments}

We thank Holger Gerhardt (Vascular Biology Laboratory, London Research Institute, Cancer Research UK, London, United Kingdom) for helpful discussions on this study and critical comments on the manuscript. We also thank Shigeyoshi Itohara (Laboratory for Behavioral Genetics, RIKEN Brain Science Institute, Saitama, Japan)

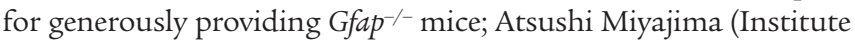
of Molecular and Cellular Biosciences, University of Tokyo, Tokyo, Japan) for preparing Lif/- mice, and Fumio Arai for technical support in the FACS study. This work was supported by Grants-in-Aid for 
Specially Promoted Research from the Ministry of Education, Culture, Sports, Science, and Technology of Japan and by a Keio University Grant-in-Aid for Encouragement of Young Medical Students.

Received for publication December 26, 2007, and accepted in revised form April 16, 2008.

1. Carmeliet, P. 2005. Angiogenesis in life, disease and medicine. Nature. 438:932-936.

2. Semenza, G.L., et al. 1999. Regulation of cardiovascular development and physiology by hypoxiainducible factor 1. Ann. N. Y. Acad. Sci. 874:262-268.

3. West, H., Richardson, W.D., and Fruttiger, M. 2005. Stabilization of the retinal vascular network by reciprocal feedback between blood vessels and astrocytes. Development. 132:1855-1862.

4. Gerhardt, H., et al. 2003. VEGF guides angiogenic sprouting utilizing endothelial tip cell filopodia. J. Cell Biol. 161:1163-1177.

5. Fruttiger, M., et al. 1996. PDGF mediates a neuron-astrocyte interaction in the developing retina. Neuron. 17:1117-1131.

6. Fruttiger, M. 2002. Development of the mouse retinal vasculature: angiogenesis versus vasculogenesis. Invest. Ophthalmol. Vis. Sci. 43:522-527.

7. Watanabe, T., and Raff, M.C. 1988. Retinal astrocytes are immigrants from the optic nerve. Nature. 332:834-837.

8. Dorrell, M.I., Aguilar, E., and Friedlander, M. 2002. Retinal vascular development is mediated by endothelial filopodia, a preexisting astrocytic template and specific R-cadherin adhesion. Invest. Ophthalmol. Vis. Sci. 43:3500-3510.

9. Stone, J., et al. 1995. Development of retinal vasculature is mediated by hypoxia-induced vascular endothelial growth factor (VEGF) expression by neuroglia. J. Neurosci. 15:4738-4747.

10. Ruhrberg, C., et al. 2002. Spatially restricted patterning cues provided by heparin-binding VEGF-A control blood vessel branching morphogenesis. Genes Dev. 16:2684-2698.
Address correspondence to: Yoshiaki Kubota or Toshio Suda, Department of Cell Differentiation, Sakaguchi Laboratory, School of Medicine, Keio University, 35 Shinanomachi, Shinjuku-ku, Tokyo 160-8582, Japan. Phone: 81-3-5363-3475; Fax: 81-3-53633475; E-mail: ykubo33@sc.itc.keio.ac.jp (Y. Kubota); sudato@sc.itc. keio.ac.jp (T. Suda).
11. Uemura, A., Kusuhara, S., Wiegand, S.J., Yu, R.T., and Nishikawa, S. 2006. Tlx acts as a proangiogenic switch by regulating extracellular assembly of fibronectin matrices in retinal astrocytes. J. Clin. Invest. 116:369-377.

12. Gough, N.M., et al. 1989. LIF: a molecule with divergent actions on myeloid leukaemic cells and embryonic stem cells. Reprod. Fertil. Dev. 1:281-288.

13. Stewart, C.L., et al. 1992. Blastocyst implantation depends on maternal expression of leukaemia inhibitory factor. Nature. 359:76-79.

14. Weber, M.A., et al. 2005. Endogenous leukemia inhibitory factor attenuates endotoxin response. Lab. Invest. 85:276-284.

15. Ash, J., McLeod, D.S., and Lutty, G.A. 2005. Transgenic expression of leukemia inhibitory factor (LIF) blocks normal vascular development but not pathological neovascularization in the eye. Mol. Vis. 11:298-308.

16. Pepper, M.S., Ferrara, N., Orci, L., and Montesano, R. 1995. LIF in vitro Leukemia inhibitory factor (LIF) inhibits angiogenesis in vitro. J. Cell Sci. 108:73-83.

17. Baluk, P., et al. 2005. Pathogenesis of persistent lymphatic vessel hyperplasia in chronic airway inflammation. J. Clin. Invest. 115:247-257.

18. Smith, L.E., et al. 1994. Oxygen-induced retinopathy in the mouse. Invest. Ophthalmol. Vis. Sci. 35:101-111.

19. Fruttiger, M., Calver, A.R., and Richardson, W.D. 2000. Platelet derived growth factor is constitutively secreted from neuronal cell bodies but not from axons. Curr. Biol. 10:1283-1286.

20. Lindahl, P., Johansson, B., Levéen, P., and Betsholtz, C. 1997. Pericyte loss and microaneurysm formation in PDGF-B-deficient mice. Science. 277:242-245.
21. Forsythe, J.A., et al. 1996. Activation of vascular endothelial growth factor gene transcription by hypoxia-inducible factor $1 . \mathrm{Mol}$. Cell. Biol. 16:4604-4613.

22. Mi, H., Haeberle, H., and Barres, B.A. 2001. Induction of astrocyte differentiation by endothelial cells. J. Neurosci. 21:1538-1547.

23. Gu, X., et al. 2002. Effects of sustained hyperoxia on revascularization in experimental retinopathy of prematurity. Invest. Ophthalmol. Vis. Sci. 43:496-502.

24. Taga, T., and Kishimoto, T. 1997. Gp130 and the interleukin-6 family of cytokines. Annu. Rev. Immunol. 15:797-819.

25. Lee, J.W., Bae, S.H., Jeong, J.W., Kim, S.H., and Kim, K.W. 2004. Hypoxia-inducible factor (HIF-1)alpha: its protein stability and biological functions. Exp. Mol. Med. 36:1-12.

26. Jeong, C.H., et al. 2007. Hypoxia-inducible factor1 alpha inhibits self-renewal of mouse embryonic stem cells in Vitro via negative regulation of the leukemia inhibitory factor-STAT3 pathway. J. Biol. Chem. 282:13672-13679.

27. Land, S.C., and Tee, A.R. 2007. Hypoxia-inducible factor 1 alpha is regulated by the mammalian target of rapamycin (mTOR) via an mTOR signaling motif. J. Biol. Chem. 282:20534-20543.

28. Gomi, H., et al. 1995. Mice devoid of the glial fibrillary acidic protein develop normally and are susceptible to scrapie prions. Neuron. 14:29-41.

29. Alon, T., Hemo, I., Itin, A., Pe'er, J., Stone, J., and Keshet, E. 1995. Vascular endothelial growth factor acts as a survival factor for newly formed retinal vessels and has implications for retinopathy of prematurity. Nat. Med. 1:1024-1028. 